\title{
The Experience of Postpartum Depression among Mothers with Withdrawn Attachment Style during Pregnancy and the First Postnatal Month
}

\author{
Mari Ikeda1 ${ }^{*}$, Momoko Hayashi², Kiyoko Kamibeppu ${ }^{3}$ \\ ${ }^{1}$ Nursing Administration and Advanced Clinical Nursing, Division of Health Science \& Nursing, Graduate School \\ of Medicine, The University of Tokyo, Tokyo, Japan \\ ${ }^{2}$ College of Contemporary Psychology, Rikkyo University, Saitama, Japan \\ ${ }^{3}$ Family Nursing, Division of Health Science \& Nursing, Graduate School of Medicine, The University of Tokyo, \\ Tokyo, Japan \\ Email: *maritake-tky@umin.ac.jp
}

Received 4 November 2015; accepted 21 November 2015; published 24 November 2015

Copyright (C) 2015 by authors and Scientific Research Publishing Inc.

This work is licensed under the Creative Commons Attribution International License (CC BY).

http://creativecommons.org/licenses/by/4.0/

(c) (i) Open Access

\section{Abstract}

Approximately $19 \%$ of women experience minor or major depression in the first three months following childbirth. Most research suggests that women with withdrawn attachment styles are less likely to be depressed. However, unlike mothers in Western culture, mothers in Japan with withdrawn attachment styles are reported to have greater potential to become depressed. Thus, the aim of this study was to describe the behaviors and situational awareness of Japanese mothers' with withdrawn attachment styles during pregnancy in order to reveal the specific dynamics underlying the withdrawn style. Interview data were analyzed using qualitative thematic content analysis. Of the 84 women assessed, 12 were determined to have a withdrawn style, and based on the Mini-International Neuropsychiatric Interview, five demonstrated the onset of postpartum depression (PPD). Two themes regarding relationships with partners emerged including: 1) confiding behavior: the importance of what is shared; and 2) need to be heard: wanting sympathy rather than criticism. Additionally, three themes describing mothers' experiences during the first postnatal month emerged: 1) overwhelming experiences: the childbirth experience; 2) seeking help: behavior changes altered relationships; and 3) experience with the baby. Withdrawn style mothers keep distance from crisis, avoid closeness with others, and opt to manage problems by themselves. However, childbirth and infant care are not easily managed in this style. Mothers who used childbirth to confide in their partners may have reduced the severity of insecurity or devel-

*Corresponding author.

How to cite this paper: Ikeda, M., Hayashi, M., \& Kamibeppu, K. (2015). The Experience of Postpartum Depression among Mothers with Withdrawn Attachment Style during Pregnancy and the First Postnatal Month. Open Journal of Depression, 4, 61-68. http://dx.doi.org/10.4236/ojd.2015.44008 
oped a more secure style. Healthcare professionals are in the best position to develop good relationships with new mothers and provide support that focuses more on emotional factors and self-esteem levels.

\title{
Keywords
}

\author{
Attachment Style Interview, Japan, Postpartum Depression, Relationship with Partner, Withdrawn \\ Style
}

\section{Introduction}

Approximately 19\% of women develop postpartum depression (PPD) in the first three months following childbirth (Gavin et al., 2005). In Japan, depression is experienced by approximately 21\% of mothers in the first month following delivery (Ikeda, Hayashi, \& Kamibeppu, 2014). Risk factors for PPD include previous depression, anxiety and depressive symptoms during pregnancy, recent stressful life events, lack of social support, and low self-esteem (Boyce \& Hickey, 2005; Milgrom et al., 2008).

Social psychology studies use a personality construct known as "attachment style" to predict depression risk (Bifulco, Moran, Ball, \& Bernazzani, 2002; Gerlsma \& Luteijn, 2000). Close relationships are important for psychological health and well-being, and attachment theory provides a critical developmental framework for understanding how individuals form close relationships, first as children and later as adults (Bowlby, 1982). Secure attachment styles involve an organized strategy, optimizing approach, or avoidance of others to find a balance of autonomy and safety/support. Bowlby's (1988) work in developmental psychology and that of cognitive and interpersonal theorists (Brown \& Harris, 1978) indicated that people with an insecure attachment style, characterized by either worry about emotional availability or support from others or by distrust or fear of closeness, selectively attend to aspects of interpersonal interactions that support their views. Insecure schemas also influence behavior and confirm this schema because people act in response to these distorted perceptions, inadvertently alienating others and undermining relationships. These cognitive schemas pertaining to relationships function largely outside of conscious awareness and can engender feelings of loss and isolation. Consequently, they may predispose these individuals to depression. While insecure attachment styles are highly associated with psychological disorders, the secure style is consistently found to be associated with the absence of disorder (Mickelson, Kessler, \& Shaver, 1997). However, Bifulco et al. (2002, 2004) reported that those with withdrawn styles were less likely to become depressed. Individuals with withdrawn style, that are one of the insecure attachment styles, can be characterized by high self-reliance, high constraints on closeness, and low desire for company. This is often expressed as desire for privacy and setting clear boundaries with others. Additionally, those with withdrawn styles prefer to rely on their own judgments in decision-making and can appear as very practical, rational, and unemotional (Bifulco \& Thomas, 2013). Behaviorally, such individuals tend to be socially isolated, which may protect them from becoming depressed (Rholes, Simpson, Campbell, \& Grich, 2001).

In our previous research examining risk factors reflecting personality structure, we found that the insecure attachment style was significantly related to PPD, and those with withdrawn styles were the most prevalent in the PPD group (Ikeda et al., 2014). The latter was a new finding that differed from results reported in studies about mothers in Western culture.

Thus, the aim of this study was to describe the behaviors and situational awareness of Japanese mothers' with withdrawn attachment styles during pregnancy in order to reveal the specific dynamics underlying the withdrawn style.

\section{Method}

\subsection{Participants}

This study was conducted at the University of Tokyo Hospital, which holds 1,217 beds conducting highly advanced medical treatment for outpatients and inpatients. The hospital received the certificate of accreditation for its hospital functions by the Japan Council for Quality Health Care. The study protocol and the assessment pro- 
cedures were reviewed and approved by the Ethics Committee of the University of Tokyo. Women were recruited at an antenatal class. Inclusion criteria were that participants were women between 28 and 32 gestational weeks pregnant, married, carrying a single fetus, and willingness to sign an informed consent release, and having a withdrawn attachment style.

\subsection{Measures}

The research was conducted between October 2010 and September 2011. Data collection involved two phrases. The first phase was completed during pregnancy. The Attachment Style Interview (ASI) (Bifulco, Lillie, Ball, \& Moran, 1998) was conducted by a trained administrator in a private interview room at either the university or the participant's home. The interviews were all recorded using an integrated circuit recorder. The ASI is a semi-structured interview that measures attachment style by gathering information about ongoing relationships with partners, family, and friends, as well as attitudes towards autonomy, closeness/distance, fear, or anger in these relationships. The interview takes about sixty minutes to administer.The sections are scored and summarized to indicate the participant's degree of insecurity and their insecurity style, which can be classified as one of the following: enmeshed, fearful, angry-dismissive, or withdrawn. All ratings were determined via consensus with a second researcher, who had no knowledge of the participants. Participants also completed a self-report questionnaire that included demographic characteristics such as age, educational level, marital status, household composition, and self-assessed socioeconomic status (SES). The second phase took place at the one-month postpartum visit. In this phase, a short interview was conducted with participants. Mothers were asked to speak freely, and interviews started with an open-ended request, "Please tell me about your experience with delivery and how your days have been during the first month postpartum". During the interview, a trained psychologist also assessed the severity of PPD symptoms using the Mini-International Neuropsychiatric Interview (M.I.N.I.) (Sheehan et al., 1998).

\subsection{Data Analysis}

We used two sources of qualitative data. The first was the verbatim ASI transcripts, a rich source of data about participants' relationship dynamics with their partners. The second qualitative data source was derived from the short interview conducted during the one-month postpartum visit.

In accordance with the thematic content analysis procedure, each transcribed interview was initially read thoroughly and notes were taken to bring out the character of the text (Braun \& Clarke, 2006).

All significant content related to confiding in others were extracted from the data. Texts were initially coded and results were placed in sub-categories that were amalgamated later in the process. Then, we sorted categories under identified themes. In order to validate analysis, the co-authors were involved throughout the entire process. Specifically, two members of the research team first separately coded the transcripts, and then met to discuss areas of consensus and discrepancy. Using a feedback loop consisting of the three researchers, the categories were revised and refined until they had been reduced to the main categories, and were subsequently rechecked. The MAXQDA version 11 (VERBIGmbH, Berlin, Germany, 2012) qualitative analysis software package was used for analysis.

\section{Results}

Of the 84 women assessed, 12 women were identified as having the withdrawn attachment style in the insecure attachment category. While no participant showed depression symptoms during pregnancy, five developed PPD at one month after delivery (depressed group), and seven did not (non-depressed group). The ages of the 12 mothers ranged from 27 to 42, and the average age was 32.8. A total of nine mothers had a university-level education and three had high-school level. Other demographic variables are shown in Table 1.

One of the major attachment behaviors measured by the ASI is confiding in others. Confiding is the extent that participants could talk about emotionally charged topics. Active emotional support from the partner is also part of the interview and reflects the extent to which the partner responded to his wife's confiding. Two themes regarding relationships with partners were identified from the data.

\subsection{Confiding Behavior: The Importance of What Is Shared}

The sub-categories related to confiding were interwoven. The non-depressed group was able to confide in their 
Table 1. Sociodemographic variables of mothers with withdrawn attachment style.

\begin{tabular}{|c|c|c|c|c|c|c|c|c|c|c|}
\hline & & \multicolumn{3}{|c|}{ Total $(N=12)$} & \multicolumn{3}{|c|}{ Depressed $(n=5)$} & \multicolumn{3}{|c|}{ Non-depressed $(n=7)$} \\
\hline & & Mean & $n$ & $\%$ & Mean & $n$ & $\%$ & Mean & $n$ & $\%$ \\
\hline Age & (Years) & 32.8 & & & 34.8 & & & 31.4 & & \\
\hline \multicolumn{11}{|c|}{ Educational level } \\
\hline & College or higher education & & 9 & $75 \%$ & & 3 & $60 \%$ & & 6 & $86 \%$ \\
\hline & Completed high school & & 3 & $25 \%$ & & 2 & $40 \%$ & & 1 & $14 \%$ \\
\hline \multicolumn{11}{|c|}{ Employment } \\
\hline & Employed & & 7 & $58 \%$ & & 2 & $40 \%$ & & 5 & $71 \%$ \\
\hline & Unemployed & & 5 & $42 \%$ & & 3 & $60 \%$ & & 2 & $29 \%$ \\
\hline \multicolumn{11}{|c|}{ Socioeconomic status (self-assessed living condition) } \\
\hline & High & & 2 & $17 \%$ & & 0 & $0 \%$ & & 2 & $29 \%$ \\
\hline & Medium & & 10 & $83 \%$ & & 5 & $100 \%$ & & 5 & $71 \%$ \\
\hline & Low & & 0 & $0 \%$ & & 0 & $0 \%$ & & 0 & $0 \%$ \\
\hline
\end{tabular}

partners about a broader range of topics more frequently than the depressed group. For example, emotional feelings related to distress associated with pregnancy and the experience of miscarriage were shared with partners in the non-depressed group. In contrast, rather than confiding about feelings, the depressed group tended to only communicate with their partners about facts.

I briefly reported details about the doctor's visit. How long it took and what the doctor said, just that... (Depressed)

All I could do was to cry for a while (after the miscarriage). He let me indulge in sorrow, while keeping his eyes on me. He cooked for me, and tried to come home early from work for me. (Non-depressed)

Additionally, the depressed group had more limitations in the topics that they could share with their partners. For instance, mothers in the depressed group were more hesitant to discuss financial problems and thoughts regarding childrearing. They also did not discuss their feelings of depression about leaving their pre-pregnancy jobs. Although mothers in the non-depressed group also regretted leaving their jobs, they avoided the topic because they accepted that this was a typical issue during the childrearing phase.

I really wanted to keep on working but I assumed he wouldn't care or understand my feelings. (Depressed)

I wanted to talk about how to raise our child and so forth. But, he seems too busy with his own stuff, but the truth is, I really wanted to talk. (Depressed)

I have nothing I cannot confide about to my husband. If there is something too minimal to discuss, I might not talk about it. Of course, if there are financial problems I am worried about, we will talk! (Non-depressed)

\subsection{Need to Be Heard: Wanting Sympathy Rather than Criticism}

Talking with partners provided mothers with a sense of security. The mothers' partners in the non-depressed group intentionally took time to listen. On the other hand, mothers in the depressed group indicated that their partners were unwilling to listen or too busy with their own lives.

He tries to listen to me even when he is busy. (Non-depressed)

When I need it, he will take time to listen no matter what. (Non-depressed)

I try not to talk about my business, my problems, because he seems so busy with his work. (Depressed)

Mothers in the depressed group experienced little sympathy and received disappointing responses from their partners. Partners of mothers in the depressed group often criticized their wives rather than providing support.

He would not listen as I wanted him to. I just want him to listen to what I would like to say... with sympathy. (Depressed)

He criticizes me for feeling depressed. It's hard to even breathe. (Depressed)

Furthermore, mothers in the depressed group often had arguments with or experienced negative attitudes from their partners. For instance, these mothers became frustrated because their partners would not listen in the de- 
sired way.

He makes me angry. We often fight. I accuse him and sometimes I've done it so much that he gets really angry too. He says he gets irritated by how I speak to him improperly and abusively. (Depressed)

In terms of mothers' experiences in the first postnatal month, three themes were identified from the data.

\subsection{Overwhelming Experiences: The Childbirth Experience}

Most mothers in the depressed group stated that the delivery was painful far beyond their expectations. Additionally, they were disappointed with how they were treated by the medical staff. For instance, they found midwives were too strict about breastfeeding education. In contrast, mothers in the non-depressed group indicated that the delivery was easy with their husbands' support.

Nobody told me that it (delivery) is this painful! (Depressed)

The midwives came too frequently to my room. They (midwives) came in a team and forced me to expel milk, even when I wanted to get a good night's rest. Everybody had the same complaints. It was almost like torture! (Depressed)

The delivery was not so bad, I was relaxed because my husband was with me through delivery. (Nondepressed)

\subsection{Seeking Help: Behavior Changes Altered Relationships}

Mothers in the depressed group did not discover anything new about their relationship with their husbands, while the mothers in non-depressed group felt that their "partner participates in childcare". For instance, one expressed that, "when the baby cries he immediately soothes the baby, it’s nice".

Mothers in both groups were concerned about their personal physical condition. For example, some had anemia or hip pain that made it challenging to fully attend to childcare. Although both groups acknowledged these conditions, their reactions differed. As a result of their withdrawn style orientation, mothers in the depressed group were unaccustomed to asking for help, leading to further depression. In contrast, mothers in the non-depressed group sought help and tried to manage their conditions.

I was hopeless. I couldn't enjoy anything at all. I can't ask for help, I've never done it before and I just don't know how. (Depressed)

I was too tired. I felt worthless all the time. (Depressed)

I became able to ask for help if it is absolutely necessary. When I think it is time to ask for help. The baby is ours and not only mine, after all. (Non-depressed)

\subsection{Experiences with the Baby}

The categories differed between the two groups in terms of their feelings toward the baby. Mothers in the depressed group reported their worries about the baby's health, feeling angry at the baby, and childcare stress. Specifically, their expectations about life with a baby did not match the reality, and they were overwhelmed with the perceived burden of childcare. On the other hand, mothers in the non-depressed group expressed their experiences as the baby is so precious, and feeling the joy of childcare.

My child is so troublesome, he doesn't even smile! He is boring. I wanted an instant toddler not a baby. (Depressed)

She cries too much no matter what I do! It is overwhelming. (Depressed)

She cries a lot and give me no break! It is never-ending workload. But that's OK, because she is so cute! (Non-depressed)

\section{Discussion}

In previous research, individuals with the withdrawn style were reported to be at low risk for PPD (Bifulco et al., 2002). Physical inconveniences during pregnancy require others' help. After the baby is born, mothers require even more assistance, which necessitates interactions with others. In the current study, individuals in the depressed group had common characteristics such as limiting the feelings they shared with their partners. Instead, they shared information about unemotional and practical issues. In contrast, those without PPD confided about emotions such as a deep sadness after the experience of miscarriage or an uncomfortable health condition. It is 
reported that the pregnancy experience has both negative and positive elements, and that the more one shares about their anxiety and worries the more positive the experience (DiPietro et al., 2004). For mothers with the withdrawn style, having a baby could be conceived as an experience of not being in control. Additionally, individuals with the withdrawn style might feel physically vulnerable in pregnancy and need support, but are impeded in attaining it because of prior independent and autonomous behavior.

Women who developed PPD did not perceive themselves as being supported emotionally, and were frustrated about not being listened to. In contrast, mothers without PPD had both emotional and instrumental support. This is important because previous research states that these are the functional elements of social support (Will \& Shinar, 2000). Emotional support can be defined as creating an environment that is pertinent to self-evaluation and fosters the experience of feeling accepted, cared for, admired, respected, and valued, despite profound personal difficulties. Individuals with the withdrawn style also pursue emotional support if they make an effort to confide in others. Negron, Martin, Almog, Balbierz, and Howell (2013) suggested that identifying new mothers' support needs and expectations is important for their recovery after childbirth. Similarly, in the current study, when participants' needs were heard and they felt supported, they did not develop PPD.

Results from the short postpartum interviews revealed that those with PPD onset had very difficult deliveries or were emotionally defeated by experiences with midwife breastfeeding training. In particular, individuals who began with a sense of self-reliance experienced a decline in self-confidence based on what they perceived as midwives' unattainable expectations. Razurel, Bruchon-Schweitzer, Dupanloup, Irion, and Epiney (2011) stated that factors associated with postpartum stress are daily hassles rather than major life events. In the early postpartum period, women reported that important stress-related factors included interactions with healthcare professionals, followed by breastfeeding and the hospital environment. Our results also suggested that those who had PPD onset expressed dissatisfaction with the support provided at both emotional and self-esteem levels. Consequently, healthcare professionals should be more aware of women's expectations in order to provide effective and adequate care during their hospital stay. In regard to relationships with infants, infants are dependent and require constant attention. Since these characteristics are in total opposition to the withdrawn style, mothers with this style were likely unable to identify infants' cues and react sensitively. For instance, Rholes, Simpson, and Friedman (2006) stated that being a parent might cause a conflict between withdrawing behavior and performing parental roles. Furthermore, Hayashi (2010) reported that individuals' with a withdrawn style kept distance from crisis, avoided closeness with others, and opted to manage problems themselves. However, as previously stated, childbirth and caring for an infant is not an easy task that can easily be managed by those with a withdrawn style. Mothers who used childbirth as an opportunity to confide in their partners may have reduced the severity of insecurity or even started to develop a more secure style. In other words, individuals who experience better quality relationships can gain satisfaction from these new relationships, which then influences their attachment style (Hammond \& Fletcher, 1991).

Individuals with a withdrawn style do not strongly desire interaction with others, which can result in a lack of dependence on healthcare professionals. Perhaps this occurs because these mothers lack the motivation, social skills, or supportive social networks necessary for seeking assistance. Simpson, Rholes, Campbell, Tran, and Wilson (2003) reported that the period immediately after delivery could be managed using the typical withdrawn style. However, withdrawn mothers soon realize that they need a tremendous amount of help and lose control over their daily activities as a result of childcare, which could lead to depression. Previous research on the withdrawn style has shown that necessary support is delayed as a result of reluctance to seek help (Feeney, 2000). In studies of individualistic Western countries, the association between avoidant attachment (including the withdrawn style discussed in this paper) and relationship problems was stronger than in more collectivist societies such as Hong Kong and Mexico (Friedman et al., 2010). There is a need to develop adequate relationships with others during the postpartum and subsequent childrearing period. Therefore, healthcare professionals should assess withdrawn style mothers' environments and provide information about the importance of integrating their partners in shared maternal interests and activities beginning in the antenatal period and continuing throughout the pregnancy (Thorp, Krause, Cukrowicz, \& Lynch, 2004). Additionally, because they meet several times during pregnancy check-ups and after childbirth, healthcare professionals are in the best position to establish a good relationship with new mothers and to reassure and encourage them about their skills. However, Bennett et al. (2009) reported that mothers were unable to find anyone in gynecologic and obstetric settings that they would feel comfortable talking to about anxiety during the postpartum period or that they felt too embarrassed to seek help. 
The significance of this study was that it used a qualitative procedure to analyze the detailed dynamics of withdrawn style mothers' relationships. Based on our findings, it is necessary to assess their resources and supports and, as with mothers with other insecure attachment styles, mothers with the withdrawn style may need close follow-up.

One limitation of the current study was the use of convenience sampling rather than a more representative sample because participants were only recruited from one hospital in an urban area in Japan. However, by conducting two thorough interviews, yielded rich contextual data that revealed information about mothers' actual relationship experiences during the perinatal period.

\section{Conclusion}

This study provides important information for healthcare professionals to consider during mothers' prenatal and follow-up care. Specifically, the findings highlight discrepancies from previous studies that present withdrawn style mothers as being at low risk for PPD. Instead, results indicate that mothers with the withdrawn style are at risk for developing PPD, and support for these women is necessary in Japan. Consequently, a thorough assessment of support availability after childbirth should be conducted. Although professionals are skilled in providing informative support, effective support should have an increased focus on emotional factors and self-esteem levels. Future research should also focus on tailored interventions that suit the types of insecure attachment in interaction, to foster a secure attachment style.

\section{Acknowledgements}

This project was supported by the Yamaji Fumiko Nursing Research Fund and Grant-in-Aid for Scientific Research (B) from the Japanese Ministry of Education, Science, Sports and Culture. We extend our gratitude to all the expectant mothers who participated in this research. We would like to thank Shiro Kozuma, PhD, MD, and Eiko Otomo, RN, for their advice and comments throughout the study. We would also like to thank Keiko Yoshida, PhD, MD for her advice regarding the Attachment Style Interview.

\section{References}

Bennett, I. M., Palmer, S., Marcus, S., Nicholson, J. M., Hantsoo, L., Bellamy, S. et al. (2009). One End Has Nothing to Do with the Other: Patient Attitudes Regarding Help Seeking Intention for Depression in Gynecologic and Obstetric Settings. Archives of Women's Mental Health, 12, 301-308. http://dx.doi.org/10.1007/s00737-009-0103-4

Bifulco, A., Figueiredo, B., Guedeney, N., Gorman, L. L., Hayes, S., Muzik, M. et al. (2004). Maternal Attachment Style and Depression Associated with Childbirth: Preliminary Results from a European and US Cross-Cultural Study. British Journal of Psychiatry (Suppl), 46, s31-s37. http://dx.doi.org/10.1192/bjp.184.46.s31

Bifulco, A., Lillie, A., Ball, B., \& Moran, P. (1998). Attachment Style Interview (ASI): Training Manual. London: Royal Holloway.

Bifulco, A., Moran, P. M., Ball, C., \& Bernazzani, O. (2002). Adult Attachment Style. I: Its Relationship to Clinical Depression. Social Psychiatry and Psychiatric Epidemiology, 37, 50-59. http://dx.doi.org/10.1007/s127-002-8215-0

Bifulco, A., \& Thomas, G. (2013). Understanding Adult Attachment in Family Relationships. Abingdon: Routledge.

Bowlby, J. (1982). Attachment (2nd ed.). New York: Basic Books.

Bowlby, J. (1988). Developmental Psychiatry Comes of Age. American Journal of Psychiatry, 145, 1-10. http://dx.doi.org/10.1176/ajp.145.1.1

Boyce, P., \& Hickey, A. (2005). Psychosocial Risk Factors to Major Depression after Childbirth. Social Psychiatry and Psychiatric Epidemiology, 40, 605-612. http://dx.doi.org/10.1007/s00127-005-0931-0

Brown, G. W., \& Harris, T. O. (1978). Social Origins of Depression: A Study of Psychiatric Disorders in Women. London: Tavistock.

Braun, V., \& Clarke, V. (2006). Using Thematic Analysis in Psychology. Qualitative Research in Psychology, 3, 77-101. http://dx.doi.org/10.1191/1478088706qp063oa

DiPietro, J. A., Ghera, M. M., Costigan, K., \& Hawkins, M. (2004). Measuring the Ups and Downs of Pregnancy Stress. Journal of Psychosomatic Obstetrics and Gynecology, 25, 189-201. http://dx.doi.org/10.1080/01674820400017830

Feeney, J. A. (2000). Implications of Attachment Style for Patterns of Health and Illness. Child: Care, Health, and Development, 26, 277-288. http://dx.doi.org/10.1046/j.1365-2214.2000.00146.x 
Friedman, M., Rholes, W. S., Simpson, J., Bond, M., Diaz-Loving, R., \& Chan, C. (2010). Attachment Avoidance and the Cultural Fit Hypothesis: A Cross-Cultural Investigation. Personal Relationships, 17, 107-126. http://dx.doi.org/10.1111/j.1475-6811.2010.01256.x

Gavin, N. I., Gaynes, B. N., Lohr, K. N., Meltzer-Brody, S., Gartlehner, G., \& Swinson, T. (2005). Perinatal Depression: A Systematic Review of Prevalence and Incidence. Obstetrics \&Gynecology, 106, 1071-1083. http://dx.doi.org/10.1097/01.AOG.0000183597.31630.db

Gerlsma, C., \& Luteijn, F. (2000). Attachment Style in the Context of Clinical and Health Psychology: A Proposal for the Assessment of Valence, Incongruence, and Accessibility of Attachment Representations in Various Working Models. British Journal of Medical Psychology, 73, 15-34. http://dx.doi.org/10.1348/000711200160273

Hammond, J. R., \& Fletcher, G. J. O. (1991). Attachment Styles and Relationship Satisfaction in the Development of Close Relationships. New Zealand Journal of Psychology, 20, 56-62.

Hayashi, M. (2010). Attachment and Adolescence. Tokyo: Misuzu Shobo.

Ikeda, M., Hayashi, M., \& Kamibeppu, K. (2014). The Relationship between Attachment Style and Postpartum Depression. Attachment and Human Development, 16, 557-572. http://dx.doi.org/10.1080/14616734.2014.941884

Mickelson, K. D., Kessler, R. C., \& Shaver, P. R. (1997). Adult Attachment in a Nationally Representative Sample. Journal of Personality and Social Psycholology, 73, 1092-1106. http://dx.doi.org/10.1037/0022-3514.73.5.1092

Milgrom, J., Gemmill, A. W., Bilszta, J. L., Hayes, B., Barnett, B., Brooks, J. et al. (2008). Antenatal Risk Factors for Postnatal Depression: A Large Prospective Study. Journal of Affective Disorders, 108, 147-157. http://dx.doi.org/10.1016/j.jad.2007.10.014

Negron, N., Martin, A., Almog, M., Balbierz, A. L., \& Howell, E. A. (2013). Social Support during the Postpartum Period: Mothers' Views on Needs, Expectations, and Mobilization of Support. Maternal and Child Health Journal, 17, 616-623. http://dx.doi.org/10.1007/s10995-012-1037-4

Razurel, C., Bruchon-Schweitzer, M., Dupanloup, A., Irion, O., \& Epiney, M. (2011). Stressful Events, Social Support and Coping Strategies of Primiparous Women during the Postpartum Period: A Qualitative Study. Midwifery, 27, $237-242$. http://dx.doi.org/10.1016/j.midw.2009.06.005

Rholes, W., Simpson, J., Campbell, L., \& Grich, J. (2001). Adult Attachment and the Transition to Parenthood. Journal of Personality and Social Psychology, 81, 421-435. http://dx.doi.org/10.1037/0022-3514.81.3.421

Rholes, W. S., Simpson, J. A., \& Friedman, M. (2006). Avoidant Attachment and the Experience of Parenting. Personality and Social Psychology Bulletin, 32, 275-285. http://dx.doi.org/10.1177/0146167205280910

Sheehan, D. V., Lecrubier, Y., Sheehan, K. H., Amorim, P., Janavs, J., Weiller, E. et al. (1998). The Mini-International Neuropsychiatric Interview (M.I.N.I.): The Development and Validation of a Structured Diagnostic Psychiatric Interview for DSM-IV and ICD-10. Journal of Clinical Psychiatry, 59, 22-33; Quiz 34-57.

Simpson, J. A., Rholes, W. S., Campbell, L., Tran, S., \& Wilson, C. L. (2003). Adult Attachment, the Transition to Parenthood, and Depressive Symptoms. Journal of Personality and Social Psychology, 84, 1172-1187. http://dx.doi.org/10.1037/0022-3514.84.6.1172

Thorp, S., Krause, E., Cukrowicz, K., \& Lynch, T. (2004). Postpartum Partner Support, Demand-Withdraw Communication, and Maternal Stress. Psychology of Women Quarterly, 28, 362-369. http://dx.doi.org/10.1111/j.1471-6402.2004.00153.x

Will, A., \& Shinar, O. (2000). Measuring Perceived and Received Social Support. In S. Cohen, L. G. Underwood, \& B. Gottlieb (Eds.), Social Support Measurement and Intervention: A Guide for Health and Social Scientists (pp. 86-135). Toronto: Oxford University Press. 